\title{
Trend of Death in Pediatric Ventriculoperitoneal Shunts in the USA
}

\author{
Kimberly H. Nguyen ${ }^{\text {a, d }}$, Hailey B. Gaskamp ${ }^{\text {a }}$, Michael E. Walston ${ }^{a}$, \\ Regina Zamacona ${ }^{\mathrm{a}}$, Jared Alexander Stowers ${ }^{\mathrm{b}}$, Ali Seifi ${ }^{\mathrm{c}}$
}

\begin{abstract}
Background: Ventriculoperitoneal shunts (VPS) have been used to treat hydrocephalus for many years. This procedure has saved many lives, but it does not come without its complications. Considering the invasiveness of the procedure, we sought to investigate the mortality rate of VPS in children in the USA.
\end{abstract}

Methods: This retrospective cohort study used the Nationwide Inpatient Sample database to examine patients from the ages of $0-17$ years who underwent the procedures involving the insertion, replacement, or removal of VPS from 1997 to 2012. Z-test was used to analyze the statistical significance.

Results: During the study period, we recorded a total number of 165,484 cases of VPS with $54.5 \%$ occurring in males. The mean age was 5.25 years old, and the average mortality rate was $1.03 \%$. The annual number of patients receiving VPS at all institutions during the study period decreased significantly from 13,075 in 1997 to 8,499 in $2012(\mathrm{P}=0.001)$. The rate of patients receiving VPS per 100,000 discharges at all institutions during the study period also decreased significantly from 18.4 in 1997 to 11.5 in $2012(\mathrm{P}=0.0001)$. The number of in-hospital deaths significantly decreased from 150 in 1997 to 50 in $2012(\mathrm{P}=0.001)$, and the main decrease in the mortality happened after $2009(\mathrm{P}=0.001)$.

Conclusions: Utilization of pediatric VPS decreased in the USA between 1997 and 2012, and the number of in-hospital deaths significantly decreased as well. This may be related to better healthcare services in recent years, especially with more emphasis on the inpatient quality indicators (IQIs). Future research should determine the cause of the significant decrease and use the information to continue bringing the mortality rate down.

Manuscript submitted March 7, 2020, accepted May 7, 2020

Published online May 30, 2020

aUniversity of Incarnate Word School of Osteopathic Medicine, San Antonio, TX, USA

${ }^{\mathrm{b} S c h o o l}$ of Medicine, UT Health San Antonio, San Antonio, TX, USA 'Division of Neurocritical Care, Department of Neurosurgery, UT Health San Antonio, San Antonio, TX, USA

${ }^{\mathrm{d}}$ Corresponding Author: Kimberly H. Nguyen, University of the Incarnate Word School of Osteopathic Medicine, 7615 Kennedy Hill Drive, San Antonio, TX 78235, USA. Email: kimberlynguyen365@gmail.com

doi: https://doi.org/10.14740/jnr573
Keywords: Ventriculoperitoneal shunts; Hydrocephalus; Pediatric

\section{Introduction}

Ventriculoperitoneal shunts (VPS) have been used to treat hydrocephalus for many years (Fig. 1) [1]. A shunt is used as a drainage system to keep fluid from the brain flowing in the right direction and at the proper rate. One end of the tube is usually placed in the ventricles while the other end enters another part of the body, where the excess cerebrospinal fluid is more easily absorbed, like the peritoneum (Fig. 2) [1, 2]. This procedure has saved many lives, but it does not come without its complications. Unfortunately, complications after VPS are common, and many revisions are needed throughout a person's lifetime. Obstruction is the most common complication, and infection is the most common complication observed in infants [3]. Considering the invasiveness of this procedure, we sought to investigate the trend of usage and the mortality rate of VPS in children in the USA.

\section{Materials and Methods}

The research was conducted retrospectively using the Healthcare Cost and Utilization Project network (HCUPnet), a database that contains data from hospitals all throughout the USA. For this research study, data were examined from patients between the ages of $0-17$ years, classified under the Clinical Classification Software Procedures code for VPS between 1997 and 2012. This is currently the latest available year for the pediatric population in the HCUPnet database. This specific code describes the insertion, replacement, or removal of extracranial ventricular shunts. This study examined the annual number of patients receiving VPS, the rate of patients receiving VPS per 100,000 discharges, and the number of in-hospital deaths. A statistical Z-test was used to infer statistical significance of the change in mortality during the years studied. Furthermore, the data were categorized according to patient demographics, specifically by gender and age. This was done to observe general patient trends and potential susceptibilities in relation to in-hospital deaths due to VPS. 


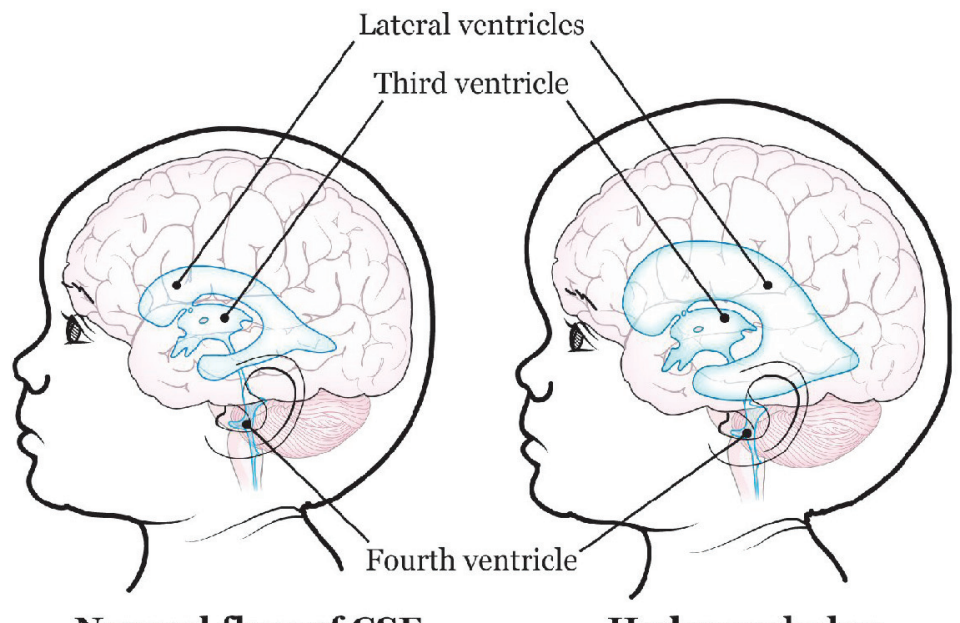

Normal flow of CSF

Hydrocephalus

• MSKCC, 2114

Figure 1. Brain without and with hydrocephalus in a child.

\section{Results}

\section{Demographics}

A total of 165,484 VPS were placed in patients between 0 and 17 years old during the study period, with a $35 \%$ decrease in the annual number of patients receiving VPS from 1997 to 2012 (Fig. 3). There was a significant decrease in number of patients discharged with VPS during the study period, with 13,074 discharges recorded in 1997 and 8,499 recorded in $2012(\mathrm{P}=0.001)$. Additionally, we found a significant decrease in the rate of discharges per 100,000 persons with VPS $(\mathrm{P}=$
0.0001). In 1997, patients in the cohort were discharged at a rate of 18.44 per 100,000 persons versus 11.53 per 100,000 persons in 2012. Within the cohort, $54.4 \%$ of the cases were male, with a mean cohort age of 5.25 years old. There was a significant increase in the mean age of the cohort during the study period, as the average age increased from 4.95 years old in 1997 to $5.61( \pm 0.14)$ years in $2012(\mathrm{P}=0.01)$.

\section{In-hospital deaths}

During the study period, there was a significant decrease in in-hospital deaths associated with VPS placement $(\mathrm{P}=0.001)$

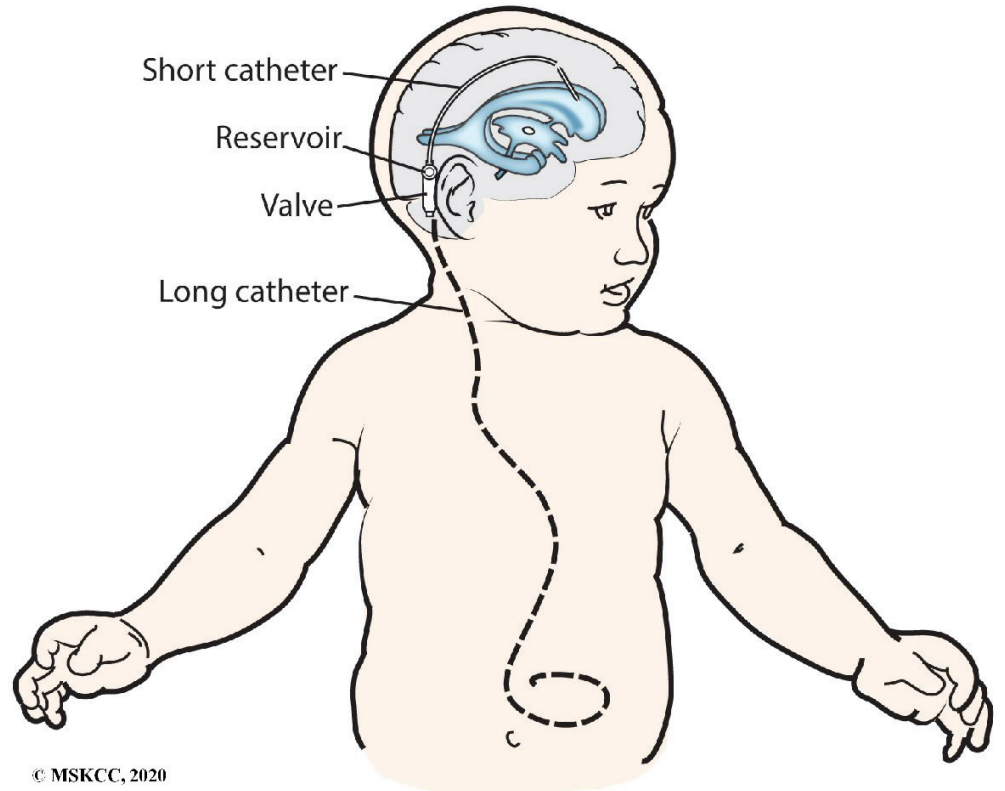

Figure 2. Ventriculoperitoneal shunt (VPS) in a child. 
Total number of discharges from 1997-2012

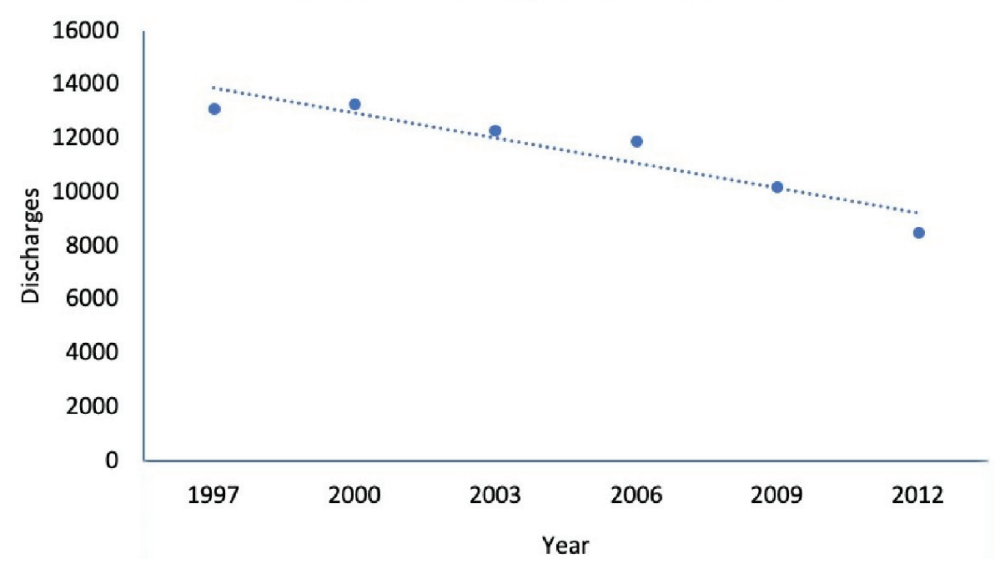

Figure 3. Total number of ventriculoperitoneal shunts between 1997 and 2012. Data were taken from the Healthcare Cost and Utilization Project network.

(Fig. 4). In 1997, there were 149.56 reported patient deaths. Mortality decreased to 49.99 ( \pm 18.87$)$ patients in 2012. This is a $67 \%$ down-trend during the study period, with the greatest decline observed after 2009. Decreases in total in-hospital deaths also correlated with a reduction in percentage of in-hospital deaths in patients with VPS $(\mathrm{P}=0.01)$. The in-hospital death percentage in 1997 was $1.14 \%$, while in 2012 it fell to $0.59 \%$.

\section{Discussion}

This study investigated the trend of usage and the mortality rate of VPS in children in the USA. These data support the increasing focus on quality improvement in healthcare and the development of systems to monitor, quantify, and incentivize quality improvement in healthcare [4]. In 2003, the Inpatient Quality Reporting Program was established and expanded the requirements for public reporting of quality data. In 2006, the
Physician Quality Reporting Initiative (PQRI) was also created to incentivize physicians and providers to report quality data, and this later transitioned into the Physician Quality Reporting System (PQRS), a program which would later transition into a penalty program [5].

The downward trend in mortality associated with VPS could very well be due to this focus on the role of quality in healthcare. With the passage of the Patient Protection and Accountable Care Act (PPACA) in 2010, two specific provisions may be related to the decrease in mortality rates of VPS. These provisions are the Patient-Centered Outcomes Research Institute (PCORI), which focuses on determining the most effective interventions based on research, and the penalty-based quality improvement provision, which prohibits federal payments to states for Medicaid services related to certain hospital-acquired infections which started in February 2011 [4]. This aligns with the sharp decrease in in-patient deaths from VPS after 2009.

However, there was a significant decrease in the annual number of patients receiving VPS from 1997 to 2012, and the

In-hospital Deaths from Ventriculo-Peritoneal Shunts from

1997-2012

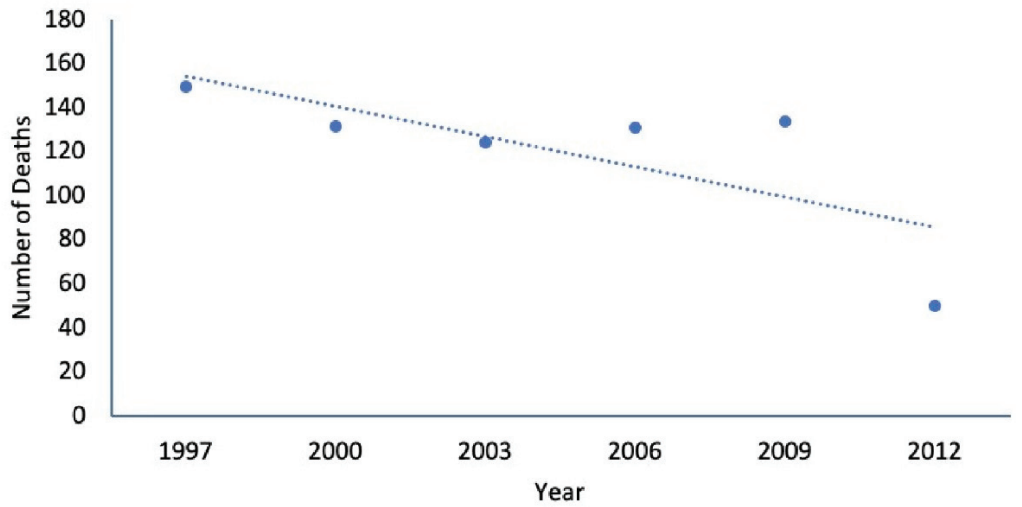

Figure 4. In-hospital deaths from ventriculoperitoneal shunts from 1997 to 2012. Data were taken from the Healthcare Cost and Utilization Project network. 
rate of patients receiving VPS per 100,000 discharges also decreased significantly. With the use of VPS on the decline, it makes sense that the number of shunt-related deaths would decrease as well. There is also a possibility that advances in the fields of biomedical engineering have decreased the number of deaths from implanted CSF shunts, allowing many patients to live somewhat normal lives [6]. As more advances continue, the incidence of death due to VPS might continue decreasing.

Though the incidence of death due to VPS is declining, shunt devices are still prone to complications. Shunt-related mortality can be encountered anytime post-surgery, and patients need to be followed for life. Shunt devices are prone to infection, and the risk of functional or mechanical shunt failure is high. Studies following patients who received shunts when they were children have shown that the majority of patients required at least one revision of their shunt in their lifetime [7]. The risk of VPS malfunction is slowly declining, but it is still a possible complication over the years [8]. Therefore, it is imperative that patients who underwent the insertion of VPS receive life-long follow-up. This can possibly help bring the mortality rate down.

\section{Conclusions}

In recent years, utilization of pediatric VPS decreased in the USA between 1997 and 2012, and the number of in-hospital deaths significantly decreased as well. This may be related to better healthcare services in recent years, especially with more emphasis on Inpatient Quality Indicators (IQIs). Unfortunately, complications after VPS such as infection and shunt malfunction are still common, and revisions are needed throughout a patient's lifetime. Future research should determine the cause of the significant decrease of in-patient deaths and use the information to continue bringing the mortality rate down.

\section{Acknowledgments}

None to declare.

\section{Financial Disclosure}

The authors declared that this study has received no financial support.

\section{Conflict of Interest}

None to declare.

\section{Informed Consent}

Not applicable.

\section{Author Contributions}

$\mathrm{KN}$ : writing the manuscript and primary analysis; $\mathrm{HG}, \mathrm{MW}$, RZ, JS: reviewing and editing the article; AS: mentor and design of the project.

\section{Data Availability}

The data supporting the findings of this study are available to the public at: https://hcupnet.ahrq.gov/\#setup.

\section{References}

1. Memorial Sloan Kettering Cancer Center (MSK). About your ventriculoperitoneal shunt surgery for pediatric patients. https://www.mskcc.org/cancer-care/patient-education/pediatric-ventriculoperitoneal-shunt-surgery (Accessed May 6, 2020 with permission from MSK communications department).

2. Hydrocephalus. Mayo Clinic, Mayo Foundation for Medical Education and Research. July 26, 2019. https:// www.mayoclinic.org/diseases-conditions/hydrocephalus/ diagnosis-treatment/drc-20373609.

3. Paff M, Alexandru-Abrams D, Muhonen M, Loudon W. Ventriculoperitoneal shunt complications: A review. Interdisciplinary Neurosurgery. 2018;13:66-70.

4. Marjoua Y, Bozic KJ. Brief history of quality movement in US healthcare. Curr Rev Musculoskelet Med. 2012;5(4):265-273.

5. Heilman Erin. The history of quality reporting - Medisolv, 2017. https://medisolv.com/academy/history-qualityreporting/.

6. Weisenberg SH, TerMaath SC, Seaver CE, Killeffer JA. Ventricular catheter development: past, present, and future. J Neurosurg. 2016;125(6):1504-1512.

7. Paulsen AH, Lundar T, Lindegaard KF. Twenty-year outcome in young adults with childhood hydrocephalus: assessment of surgical outcome, work participation, and health-related quality of life. J Neurosurg Pediatr. 2010;6(6):527-535.

8. Paulsen AH, Lundar T, Lindegaard KF. Pediatric hydrocephalus: 40-year outcomes in 128 hydrocephalic patients treated with shunts during childhood. Assessment of surgical outcome, work participation, and health-related quality of life. J Neurosurg Pediatr. 2015;16(6):633-641. 